\title{
Intracellular Characterization of Song-Specific Neurons in the Zebra Finch Auditory Forebrain
}

\author{
Michael S. Lewicki \\ Computation and Neural Systems Program, California Institute of Technology, Pasadena, California 91125
}

\begin{abstract}
Auditory neurons in the forebrain nucleus HVc (hyperstriatum ventrale pars caudale) are highly sensitive to the temporal structure of the bird's own song. These "song-specific" neurons respond strongly to forward song, weakly to the song with the order of the syllables reversed, and little or not at all to reversed song. To investigate the cellular mechanisms underlying these responses, in vivo intracellular recordings were made from adult HVc neurons. Song-specific cells could be divided into those that responded strongly throughout autogenous song (tonic cells) and those that responded with bursts of action potentials at specific points during the song (phasic cells). Phasic cells were hyperpolarized during autogenous song, even though this stimulus also elicited the strongest response. Less hyperpolarization was seen to the same song with the syllables in reverse order, and none was seen to reversed song. The responses of both types of song-specific
\end{abstract}

cells contained high-frequency bursts of action potentials. The bursts of the phasic cells showed attenuation of the action potential height and lack of full repolarization after each spike. This type of bursting was significantly correlated with the amount of hyperpolarization before each burst in phasic cells and nonauditory cells that generated such bursts spontaneously. These data suggest that song-specific neurons use longlasting hyperpolarization as a mechanism to integrate auditory context, an important component of temporal order selectivity. Hyperpolarization also may increase the precision of spike timing, which could be important for the neural code subserving song learning and production.

Key words: auditory response properties; burst firing; hyperpolarization; neural integration; context sensitivity; order sensitivity; song system; HVc; intracellular recording
Neurons in the songbird forebrain nucleus HVc (hyperstriatum ventrale pars caudale, also called high vocal center) are highly sensitive to the acoustic structure in the bird's own (autogenous) song. These "song-specific" neurons respond most strongly to autogenous song, less to songs from the same species, and little or not at all to songs of other species (Margoliash, 1983, 1986; Margoliash and Konishi, 1985).

$\mathrm{HVc}$ is at an intersection of auditory and motor pathways in the songbird forebrain. It is a high-level auditory area, and there are at least two stages of post-thalamic processing before auditory information arrives at HVc (Kelley and Nottebohm, 1979; Fortune and Margoliash, 1995; Vates, 1996). HVc also is part of the motor pathway controlling song production (Nottebohm et al., 1976; McCasland, 1987; Vu et al., 1994). Song-specific neurons in HVc and other auditory areas in the song system emerge during song learning (Doupe and Konishi, 1992; Volman, 1993); thus, they are likely to play some role in that process. Because song learning is critically dependent on auditory feedback (Konishi, 1965), it is important to understand the auditory response properties of these cells.

Part of the sensitivity of song-specific neurons derives from their sensitivity to the song's temporal structure. Their response is greatly reduced if the song is reversed or if the order of the song

\footnotetext{
Received March 26, 1996; revised June 14, 1996; accepted June 25, 1996.

This work was supported by a National Institutes of Health Research Training grant and a Caltech Engineering Research Center fellowship. I thank Allison Doupe, Rich Jeo, Mark Konishi, Jamie Mazer, and Marc Schmidt for valuable comments on this manuscript.

Correspondence should be addressed to Dr. Michael Lewicki at his present address: The Salk Institute, Computational Neurobiology Lab, 10010 North Torrey Pines Road, La Jolla, CA 92037.

Copyright (C) 1996 Society for Neuroscience $0270-6474 / 96 / 165854-10 \$ 05.00 / 0$
}

syllables is reversed (Margoliash, 1983; Margoliash and Fortune, 1992; Lewicki and Arthur, 1995). Studies presenting syllables in isolation have shown that these neurons can integrate auditory information over hundreds of milliseconds (Margoliash, 1983; Margoliash and Fortune, 1992; Lewicki and Arthur, 1995).

Although song-specific neurons have been well studied with extracellular methods, little is known about the cellular mechanisms that underlie their response properties. One possibility is that song-specific cells preserve auditory information through the activation of cellular or synaptic currents. Experiments in $\mathrm{HVc}$ brain slices have suggested several possibilities. Kubota and Saito (1991) found Na-dependent outward conductances in HVc cells that persisted for several seconds. Vu and Lewicki (1994) found active glutamate receptors of both the NMDA and AMPA/kainate subtype as well as active GABAergic receptors. Schmidt and Perkel (1995) reported evidence of synaptically activated GABA and $\mathrm{GABA}_{\mathrm{B}}$ receptors. It is not known, however, what role these mechanisms play during an auditory response.

To date, there have been very few intracellular studies of $\mathrm{HVc}$ cells in vivo (Katz and Gurney, 1981; Fortune and Margoliash, 1994; Lewicki and Konishi, 1995). Song-specific neurons are difficult to study, because only $1 / 3$ of the neurons in $\mathrm{HVc}$ are auditory (the rest are presumably motor) and, of these, only half are found to be song-specific (Margoliash, 1983; Margoliash and Fortune, 1992; Yu and Margoliash, 1995). These problems are compounded with intracellular methods with which it is very difficult to obtain stable recordings. We report here, however, that stable intracellular recordings can be made from song-specific neurons. These data reveal several new properties that may be important for the neural code of these cells and suggest mecha- 
nisms by which they could integrate long periods of auditory context.

Other results from this study were reported in Lewicki and Konishi (1995).

\section{MATERIALS AND METHODS}

Experiments were performed on adult $(>120$ d) male zebra finches (Taeniopygia guttata) raised in our own colony. Before each experiment, the bird's own song was recorded, digitized, and analyzed on a computer using custom software (written by M. Lewicki, L. Proctor, and J. Mazer). A few days before the experiment, birds were anesthetized with Equithesin (0.03-0.04 ml, i.m.) (0.85 gm chloral hydrate, $0.21 \mathrm{gm}$ pentobarbital, $0.42 \mathrm{gm} \mathrm{MgSO}_{4}, 2.2 \mathrm{ml} \mathrm{100 \%}$ ethanol, $8.6 \mathrm{ml}$ propylene glycol, filled to a total volume of $20 \mathrm{ml}$ with water), and a small metal post that immobilized the head during physiological recordings was cemented to the skull with dental cement. For physiological recordings, the birds were anesthetized with urethane (65-90 $\mu$ l of a $20 \%$ solution).

Nucleus HVc was first located physiologically with extracellular glass electrodes. Electrodes were lowered through a small hole $(0.3 \mathrm{~mm}$ diameter) in the skull to minimize brain edema and pulsation. Intracellular recordings were obtained with sharp electrodes $(60-100 \mathrm{M} \Omega$, filled with $4 \mathrm{M}$ potassium acetate, $\mathrm{pH} 7.4$ ) or whole-cell patch electrodes (6-12 $\mathrm{M} \Omega$, filled with solution containing (in mM): $140 \mathrm{~K}$-gluconate, 10 HEPES, 4 $\mathrm{MgCl}_{2}, 0.1 \mathrm{CaCl}_{2}, 1.1$ EGTA, $3 \mathrm{Na}_{2}-\mathrm{ATP}, 2 \mathrm{Na}-\mathrm{GTP}, \mathrm{pH} 7.4$, and adjusted to $300-330 \mathrm{mOsm})$. Both intracellular and patch electrodes were pulled on a Flaming-Brown model P-87 micropipette puller (Sutter Instruments, Navato, CA). In some experiments, $1.75 \%$ biocytin was added to stain the cells. Intracellular potentials were amplified with an Axoclamp 2A amplifier (Axon Instruments, Foster City, CA), filtered at $10 \mathrm{kHz}$, and digitized at a sampling rate of $32 \mathrm{kHz}$ for computer analysis. Cells that did not have a stable resting potential for $>2 \mathrm{~min}$ or had an action potential height $<40 \mathrm{mV}$ were not analyzed.

Some of the stimuli used in these experiments involved manipulations of the order of syllables taken from the bird's own song. Syllable boundaries were defined as points where the song's amplitude falls to zero. The stimuli (autogenous song and its manipulations) were presented in freefield conditions with a calibrated speaker (JBL, Northridge, CA) in a custom-made sound attenuation chamber (Industrial Acoustics, Bronx, NY). The peak amplitude of the stimuli was between 60 and $70 \mathrm{~dB}$ sound pressure level.

A song-specific cell was defined as a neuron that responded significantly more to forward song than to either reversed song or the song syllables presented in reverse order. Significance was determined with a two-tailed, paired $t$ test comparing the spikes rates on a trial-per-trial basis, after subtracting the spontaneous rate for each stimulus. The spontaneous spike rate was measured during the 1-2 sec prestimulus interval. The stimulus spike rate was defined as the number of spikes produced during the stimulus divided by the duration of the stimulus. A response latency of $20 \mathrm{msec}$ was included in the analysis but in practice, this had no effect on the results. This procedure can underestimate the response if the instantaneous spike rate varies during the song. In practice, this only a problem for cells that produced bursts of action potentials at specific points during the song. Therefore, a cell also was classified as song specific if it showed a significantly different number of action potential bursts during forward song compared with reverse song. A burst was defined as a sequence of at least two action potentials in a period of $30 \mathrm{msec}$ with a maximum interspike interval $<6 \mathrm{msec}$.

The anatomical location of the recordings were determined by making reference marks made by electrolytic lesions using extracellular tungsten electrodes (AM systems, Everett, WA) and by filling the single neurons with biocytin. At the end of the experiment, the bird was perfused with saline followed by $4 \%$ paraformaldehyde for histological analysis. Electrolytic lesions were located on $30 \mu \mathrm{m}$ frozen sections stained with cresyl violet. Brains containing biocytin-filled neurons were cut into $60 \mu \mathrm{m}$ frozen sections. These were incubated for $2 \mathrm{hr}$ in $0.1 \mathrm{M}$ PBS containing avidin-biotin complex (ABC Elite, Vector Labs, Burlingame, CA) (1: $100)$ and $0.3 \%$ Triton $X-100$. Sections then were treated with $0.05 \%$ DAB, $0.005 \% \mathrm{H}_{2} \mathrm{O}_{2}, 0.005 \% \mathrm{CoCl}_{2}$, and $0.005 \% \mathrm{NiNH}_{3}$ for $5 \mathrm{~min}$. Sections were washed thoroughly $(3 \times 10 \mathrm{~min})$ in $0.1 \mathrm{M}$ PBS before each step and again at the end. All sections were dehydrated, cleared in xylene, and coverslipped with Permount.

\section{RESULTS}

Stable intracellular recordings were obtained from 97 cells in 32 birds. The mean duration of intracellular recording time was 16 $\min$. The mean initial resting potential was $-61 \pm 9 \mathrm{mV}$, and the mean action potential height was $58 \pm 13 \mathrm{mV}$. Of the 97 cells, 29 showed some auditory response, and 6 of these, in three different birds, were classified as song-specific cells (see Materials and Methods). Because of short intracellular recording times compared with extracellular recording, it is likely that the proportion of the auditory cells that were song specific is less than that reported in previous studies. Also, the response of the cell often deteriorates over time, probably as a result of damage from the electrode. The responses of the six song-specific cells reported here, however, were stable throughout the entire recording time. Song-specific cells showed no apparent differences from other cells in terms of their resting potential, action potential shape, or holding times. All the song-specific neurons were recorded with sharp intracellular electrodes.

Extracellular studies have shown that song-specific neurons can have both tonic and phasic song responses, which often contain bursts of action potentials (Margoliash and Fortune, 1992; Sutter and Margoliash, 1994). These properties also were found to be present in the intracellular records of song-specific cells. Three of the six song-specific cells were tonically excited throughout the song. The other three song-specific cells responded with rapid bursts of action potentials at specific points during the song.

\section{Tonic excitation}

Figure 1 shows an intracellular recording of a song-specific $\mathrm{HVc}$ cell that is tonically excited throughout much of the autogenous song. The response also contains many action potential bursts, which is characteristic of $\mathrm{HVc}$ neurons. The median membrane potential (which is less influenced by action potentials than by the waveform average) shows that there is some hyperpolarization (indicated by the arrows) after the forward song (Fig. 1a) and after the middle of the syllable-reversed song (Fig. 1b). No such hyperpolarization is present in the response to the reversed song (Fig. $1 c)$. The response to both the reversed and syllable-reversed song is significantly less than the response to the forward song $(p<$ 0.01 , paired $t$ test).

Another tonically excited cell also was depolarized throughout most of the forward song (data not shown). That cell showed no hyperpolarization during the syllable-reversed song, but was simply less depolarized. It also had little depolarization to the reversed song.

\section{Phasic excitation}

An example of a song-specific cell that was phasically excited is shown in Figure 2. This cell was recorded from the same bird as the neuron in Figure 1. In this paper, a phasic response refers to excitation at specific points during the stimulus. This is in contrast to the tonically excited cells discussed in the previous section, which are excited throughout the stimulus, but show little or no regularity in the temporal positions of the action potentials. The phasic response to the forward song is not present in the response to either syllable-reversed song or the reversed song. Note also that only the response to forward song contains consistent hyperpolarizations, which can be seen in the overlaid waveform rasters (Fig. 2a, bottom traces). Although this cell responded with approximately equal numbers of action potentials for all three stimuli, there were significantly more action potential bursts in response to forward song than to either the syllables in reverse order $(p<$ 


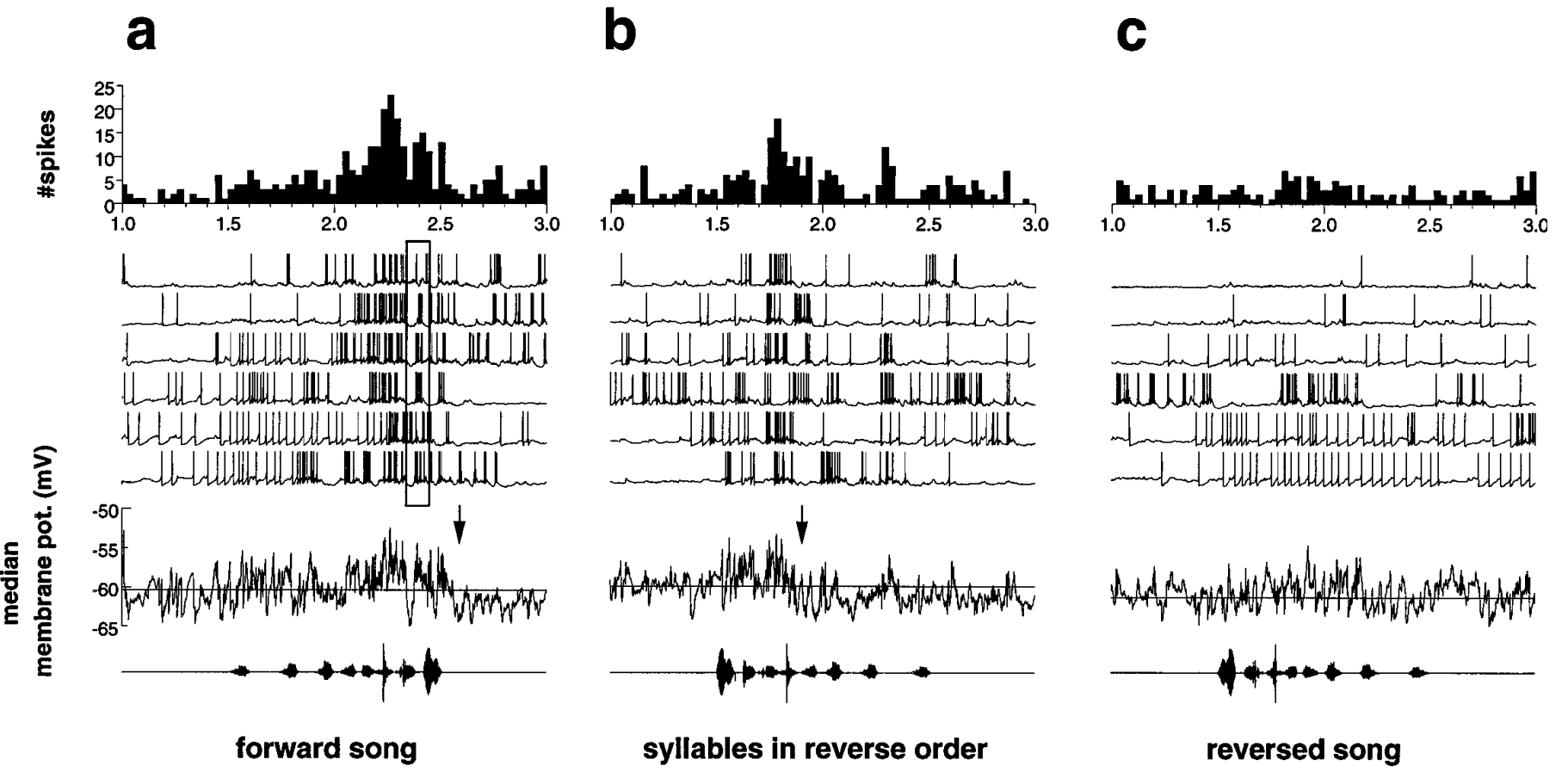

Figure 1. An intracellular recording of a tonically excited song-specific HVc cell. $a-c$ show the peristimulus time histogram (top). The traces below show the intracellular membrane potential for six collections (top traces). The collections for each stimulus were interleaved. The traces inside the box are plotted in Figure 5. Below each set of waveform rasters is the median of the individual traces (bottom trace); the average resting membrane potential is shown by the horizontal line. The oscillogram of the stimulus is plotted below (bottom). The response to the forward song $(a)$ is greater than to the song with the syllables in reverse order $(b)$ and to the reverse song $(c)$, indicating that this is a song-specific cell. The median membrane potential shows that the cell is hyperpolarized after the forward song and during the second half of the song with the syllables in reverse order $(a, b, a r r o w s)$. The cell shows no hyperpolarization to the reversed song $(c)$.

0.05 , paired $t$ test) or to the reversed song $(p<0.001)$. The bursts to forward song are outlined by the boxed region in Figure 2.

Figure 3 shows the response of another phasically excited HVc cell. This was classified as a song-specific neuron, because it responded to the forward and syllable-reversed song, but showed no response to the reversed song. The traces in Figure 3 are in response to the syllable-reversed song. The spike bursts are aligned to within $5 \mathrm{msec}$, and the response even shows remarkable consistency in the variation of the subthreshold membrane potential.

\section{Hyperpolarization during phasic excitation}

The induction of long-lasting hyperpolarizing currents after highfrequency firing has been reported from in vitro studies of $\mathrm{HVc}$ (Kubota and Saito, 1991). Similar currents were observed in vivo in response to presentation of the bird's own song. Figure 4 shows that the amount of hyperpolarization is greatest during forward song when the cell's response is strongest (Fig. 4a). The membrane potential is less hyperpolarized when the order of the syllables is reversed (Fig. 4b) and is not hyperpolarized at all in response to the reversed song (Fig. $4 c$ ). This hyperpolarization also is long lasting; the recovery to the average resting potential after the end of the forward song takes several hundred milliseconds. Long-lasting afterhyperpolarizations also could be evoked in nonauditory neurons (data not shown) with strong $(0.75 \mathrm{nA})$ current injections.

\section{Bursting during tonic and phasic excitation}

Some HVc cells were capable of firing three or more action potentials in a single, high-frequency burst. Bursting was present in both tonically and phasically excited song-specific cells. The detailed structure of the responses from the boxed regions of Figures 1 and 2 is shown in Figure 5. Both responses show bursts of action potentials, but those in Figure $5 b$ are consistently attenuated over the course of the burst, whereas no attenuation is evident in the bursts in Figure $5 a$. This attenuation can be quite dramatic as in Figure 3, where the last spike in each burst is about half the height of the first. The spike bursts in the phasic responses also were more precisely timed than those in the tonic responses. The bursts in Figure $5 a$ show no temporal alignment, but those in Figure $5 b$ temporally aligned to within $8 \mathrm{msec}$. These bursts also show consistent previous hyperpolarization. The average membrane potential in the $50 \mathrm{msec}$ period before the first spike in each burst is $6.8 \pm 2.1 \mathrm{mV}$ below average resting potential $(-68 \mathrm{mV})$. All of the phasically excited cells showed a similar pattern of bursting: lack of full repolarization and attenuation of the action potentials in addition to hyperpolarization before each spike burst.

Bursting also can occur at multiple times during the song. One such cell is shown in Figure 6. Bursting occurs most frequently to forward song where the bursts are phase locked to a particular syllable that occurs three times during the song (Fig. 6a, arrows). For this cell, the bursting is less regular than in the previous examples, but like the response shown in Figure 2, bursting is less frequent when the order of the syllables is reversed (Fig. 6b), and no bursts are present during presentation of the reversed song (Fig. 6c). This cell also bursts most frequently when it is hyperpolarized, which is shown in Figure 7.

The correlation between bursting and hyperpolarization can be analyzed additionally by comparing the prepotential, or the average membrane potential before a spike, with the number of 
a
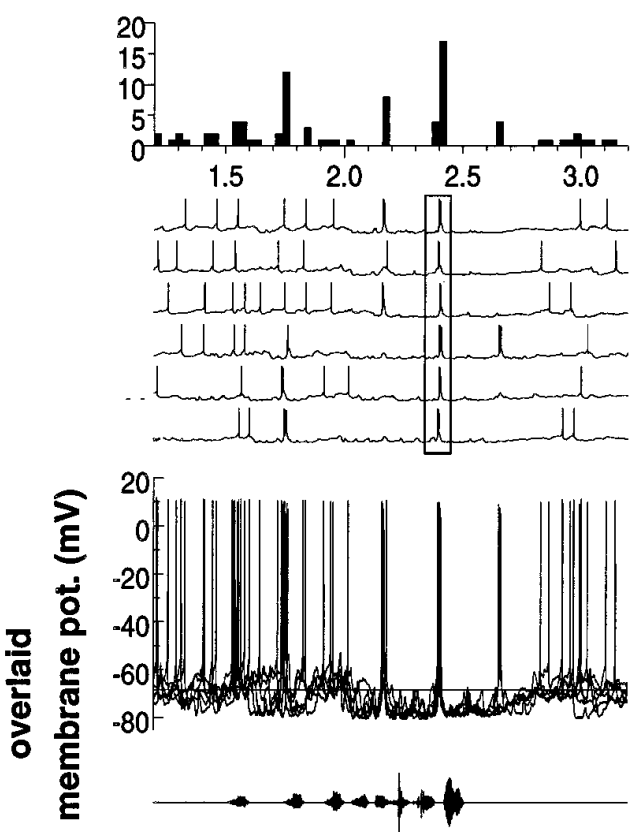

forward song b
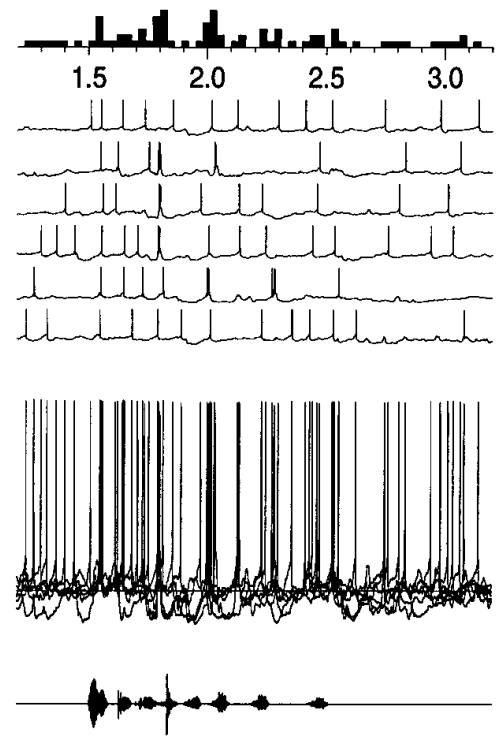

syllables in reverse order
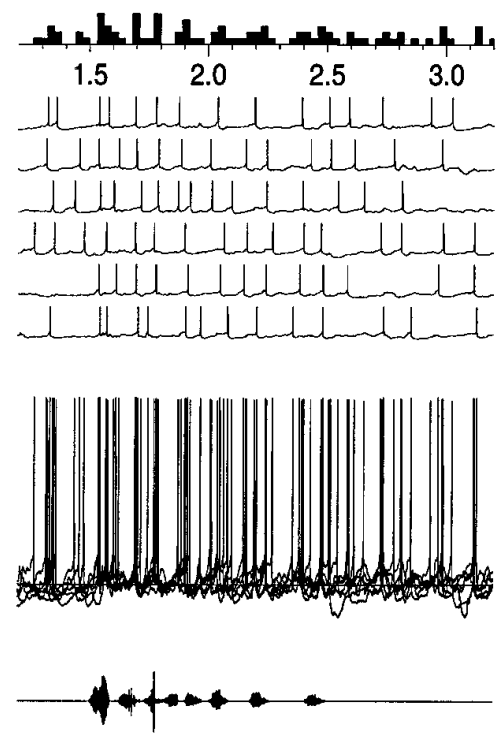

reversed song

Figure 2. An intracellular recording of a phasically excited song-specific HVc cell. The conventions are the same as in Figure 1, except that in bottom traces $(a-c)$, the waveform rasters are overlaid to show the consistency of both the hyperpolarizations and of the temporal positions of the phasic bursts. The boxed region indicates where this cell responded phasically with bursts of action potentials to the forward song. The phasic response is lost when the syllable order is reversed or when the entire song is reversed.

subsequent spikes. To examine this relationship, the action potentials in response to auditory stimuli (during stimulus and background periods) were sorted according to the prepotential, defined here as the average potential in the $50 \mathrm{msec}$ epoch before each spike relative to the average resting membrane potential. Spikes occurring within $30 \mathrm{msec}$ of an already considered spike were excluded. The prepotential and number of following spikes were measured for three groups of neurons: song-specific cells that had a phasic response to the forward song $(n=3)$, songspecific cells that had a tonic response throughout the forward

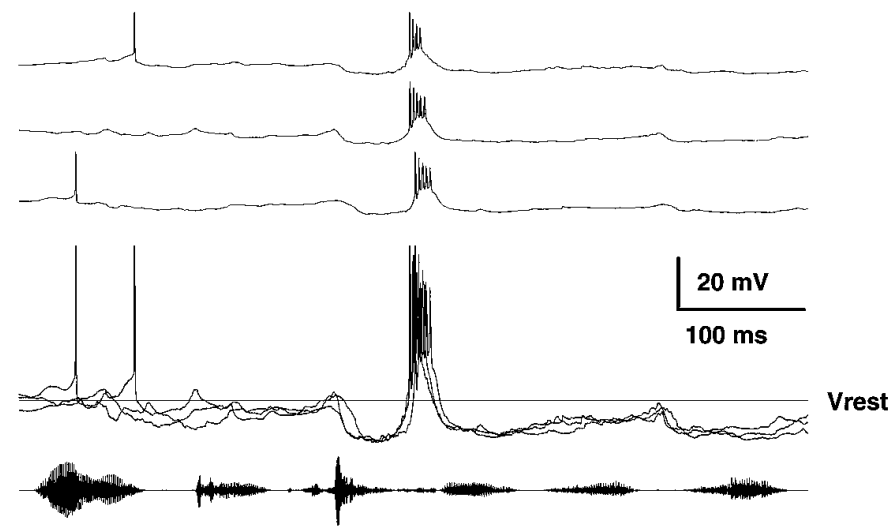

Figure 3. Precise timing in a phasically excited $\mathrm{HVc}$ cell. The horizontal line indicates the average resting potential, which was $-59 \mathrm{mV}$. Some phasic cells show high regularity in the firing times of the bursts and in the subthreshold membrane potential. The action potentials in these bursts also show attenuation over the time course of the burst. song $(n=3)$, and nonauditory cells that showed spontaneous bursting $(n=5)$. One-way ANOVA was performed for each group to determine whether there was a statistically significant change in prepotential for different numbers of subsequent spikes.

Figure 8 summarizes the distributions of prepotentials before each spike or spike burst for the three different groups. Songspecific neurons that responded phasically to song were significantly hyperpolarized before each action potential ( $p<0.001, F$ test) (Fig. 8, left), but tonically excited song-specific cells showed no significant change in prepotential versus the number of following spikes ( $p>0.5, F$ test) (Fig. 8, middle). The spike bursts produced by the nonauditory cells appear similar to those of the phasically excited song-specific cells and, like those cells, also showed a significant hyperpolarization before bursting compared with the prepotential for a single spike ( $p<0.001, F$ test) (Fig. 8 , right).

Examples of the trend suggested by this analysis can be seen using data taken from one of the phasically excited song-specific cells (the one shown in Fig. 6). The waveform patterns following the 10 most negative prepotentials of the data collected in response to the forward song are shown in Figure $9 a$. Nearly every trace is followed by a burst. The most positive prepotentials, however, are all followed by single action potentials (Fig. 9b). The other two phasically excited song-specific cells showed similar patterns.

Conversely, one can examine the membrane potential before a burst containing a certain number of spikes. Figure $10 a$ shows four waveforms aligned on the first action potential of bursts containing six spikes. These are preceded by a consistent hyperpolarization, whereas the bursts containing only three spikes are not (Fig. 
a

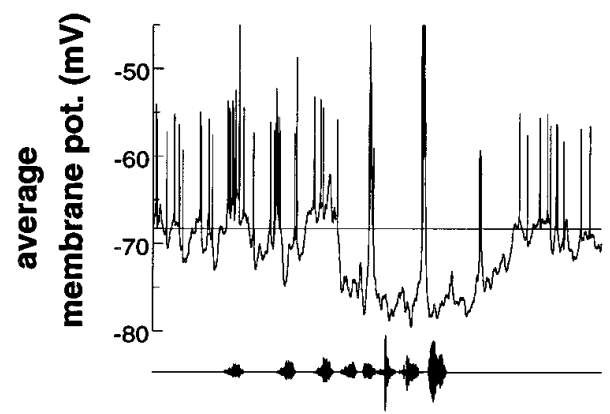

forward song b

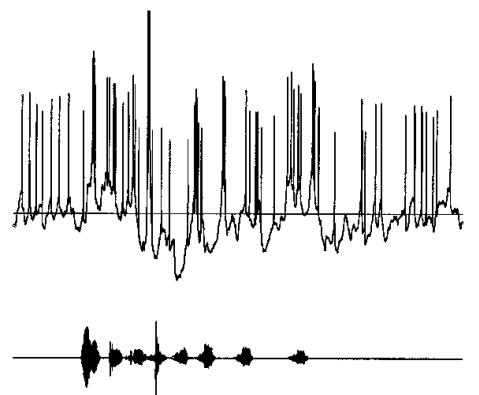

syllables in reverse order
C
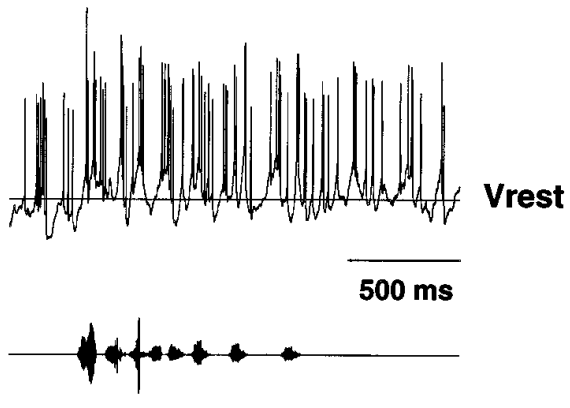

reversed song

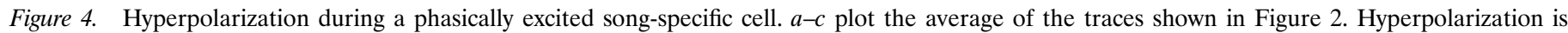
greatest during the forward song, less when the syllables are presented in reverse order, and not present when the song is reversed.

10b). These spikes are taken from the same data set used in Figure 9. The patterns seen in the membrane potential before bursting was similar in all three phasically excited song-specific cells.

\section{Morphological description and projections of HVc cells}

Three cells were successfully filled with biocytin, stained with avidin-HRP, and also had clear axonal projections that could be traced to their targets (Fig. 11). One of these cells (Fig. 11a) was song-specific (data shown in Fig. 2) and sensitive to temporal combinations of song syllables (see Lewicki and Konishi, 1995). The cell had a soma diameter of $\sim 15 \mu \mathrm{m}$, thin, spinous dendrites, and a dendritic arborization of $\sim 125 \mu \mathrm{m}$. It had a clear axonal projection to area $\mathrm{X}$.

The cells shown in Figure $11 b$ and $c$ had clear projections to the robust nucleus of the archistriatum (RA). Neither cell had an auditory response. Both cells had similar morphology and in each, the somatic diameter was $\sim 15 \mu \mathrm{m}$, and the diameter of their dendritic arborization was $\sim 100 \mu \mathrm{m}$. a

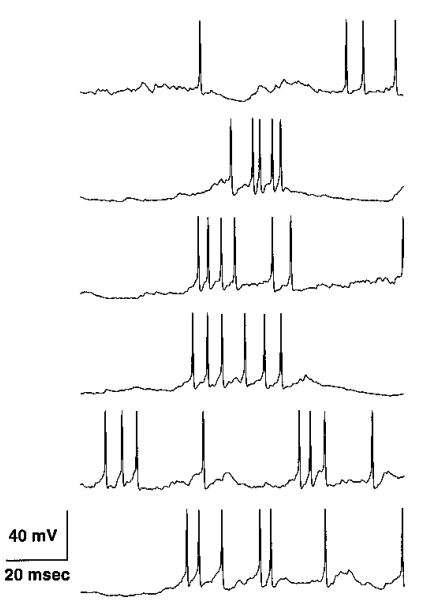

b

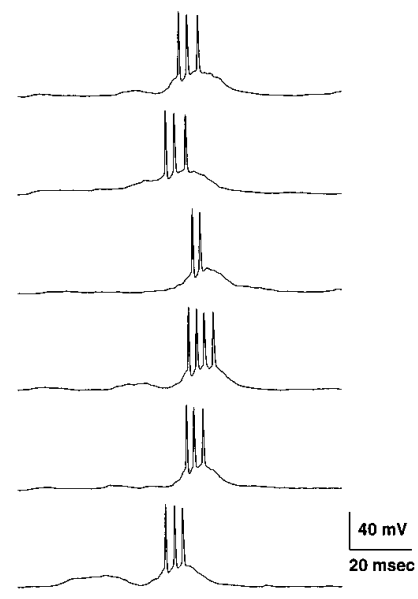

Figure 5. Comparison of burst firing in phasic and tonic song-specific cells. Traces are from the boxed regions of the tonically excited songspecific cell in Figure $1(a)$ and the phasically excited song-specific cell in Figure $2(b)$. Both responses contain bursts of action potentials, but the bursts from the phasic cell are temporally aligned and there is consistent hyperpolarization before each burst. Also, these bursts show a consistent attenuation of action potential height, which is not seen in the bursts of the tonically excited cell.

\section{DISCUSSION}

\section{Hyperpolarization during song}

Perhaps the most surprising result here is that some song-specific cells are hyperpolarized during forward song, to which they also have the greatest response. This hyperpolarization was accompanied by phasic bursts of action potentials in all three cases observed. The reason for the hyperpolarization is unclear, but one possibility is to increase the reliability of spike timing. Mainen and Sejnowski (1995) have reported that neurons spike more reliably when starting from a hyperpolarized state than from rest. There can be high regularity in the temporal alignment of phasic bursts and even in the subthreshold membrane response. Precise timing is likely to be an important aspect of both the auditory and motor code of the song system. Hyperpolarization may subserve the creation and/or preservation of this neural code.

Another possible function of the hyperpolarization could be to ensure that the neuron does not spike at inappropriate times and only spikes when there is a large depolarizing input. In this sense, a cell that is hyperpolarized would increase its signal-to-noise ratio during the song. This hypothesis predicts that depolarization of the membrane potential of a phasic song-specific cell in response to the song should decrease the reliability of spike timing.

The cells that were hyperpolarized to forward song all showed less hyperpolarization to syllable-reversed song and little or none to reversed song. This observation underscores the unique nature of song-specific neurons and their sensitivity to the temporal structure of the autogenous song. It also suggests a mechanism by which these cells can integrate long periods of auditory context. Because time constant of the hyperpolarization is several hundred of milliseconds, acoustic cues that could induce it could have a long-lasting effect on the state of the cell. These data suggest that hyperpolarization may be one of the mechanisms underlying syllable order sensitivity.

The data presented here that the response to forward song sometimes was followed by a long-lasting hyperpolarization that could outlast the stimulus by several hundred milliseconds. Longlasting hyperpolarizing currents also have been observed in $\mathrm{HVc}$ brain slices in response to high-frequency firing (Kubota and Saito, 1991). And similar long-lasting inhibition also has been seen in extracellular studies, even when the response occurs largely at the beginning of the song (Sutter and Margoliash, 1994). Data from the slice experiments of Kubota and Saito (1991) suggest that this hyperpolarization after song is a long-lasting 
a

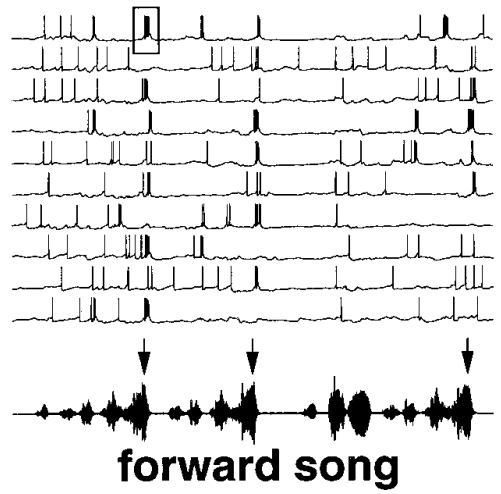

b
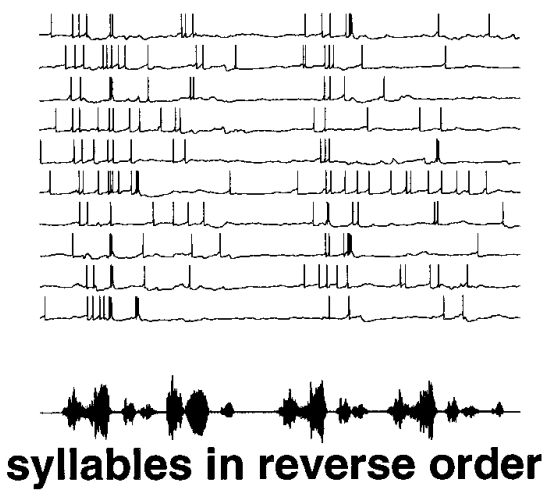

C

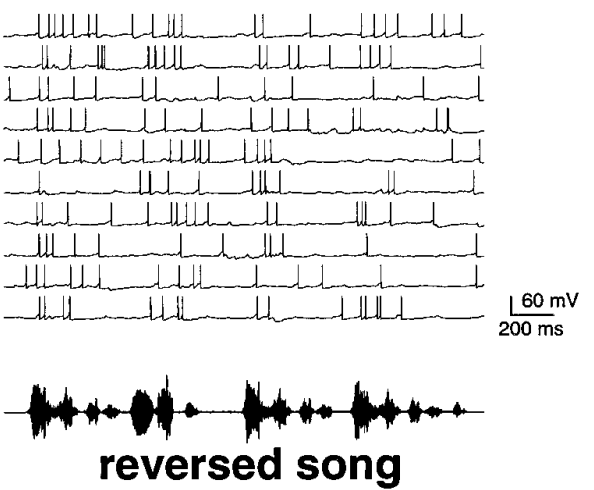

Figure 6. Burst firing during song stimuli. $a-c$ show the intracellular waveform raster of a song-specific HVc cell. The collections were interleaved. $a$, The intracellular record shows that this cell bursts (one such burst is outlined by the box) regularly after the same syllable in the forward song (arrows). $b$, Less bursting is seen when the syllables are presented in reverse order; $c$, no bursting is seen when the song is reversed.

outward current elicited by rapid firing of action potentials. The hyperpolarization seen in Figure 4, however, is present, even though there are about the same number of previous spikes as the reversed song to which there is no hyperpolarization. One explanation for these observations is that the hyperpolarization has several components and some may be synaptically driven, possibly by persistent inhibition mediated by GABAergic channels. Another possibility is that part of the hyperpolarization results from the activation of metabotropic glutamate or serotonin receptors (Schmidt and Perkel, 1995). Manipulation of the membrane potential with current injection during presentation of the song may provide data to distinguish among these hypotheses.

\section{Bursting}

The significance of the bursting accompanied by the hyperpolarization is unclear. One possibility is that these bursting neurons are similar to the mammalian thalamocortical relay neurons (Jahnsen and Llinas, 1984; Steriade and Deschenes, 1984), which also have two modes of firing, but other intracellular studies suggest HVc neurons are different, because bursting is of the high-threshold type and cannot be elicited by previous hyperpolarization (Kubota and Saito, 1991; Lewicki and Konishi, 1995). Another possible role for bursting neurons is that the bursts of action potentials may help ensure precise firing of efferent cells. Precisely timed spiking is a sensible code for the motor program underlying song production, because muscle movements required to produce song also must be precisely timed. Birds require auditory feedback to learn to produce normal song (Konishi, 1965), thus, it is plausible that the feedback also must be precisely timed.

Because song-specific cells in HVc arise during vocal learning (Volman, 1993), it is possible that these cells play a role in song learning. High-frequency bursts are capable of eliciting longlasting changes in synaptic strength (Larson et al., 1986). This plasticity depends on the activation of NMDA receptors (Kirkwood et al., 1993; Malenka, 1994), which have been shown to be present in HVc (Vu and Lewicki, 1994) and in RA (Mooney and Konishi, 1991; Mooney, 1992). The generation of action potential bursts may induce activity-dependent changes in synaptic strength within HVc or in the efferent nuclei RA and area X.

\section{HVc cell anatomy}

The results from the intracellular staining are consistent with Katz and Gurney (1981) who reported that HVc auditory neurons project to area $\mathrm{X}$ and nonauditory neurons project to RA. With such small numbers, they do not rule out the possibility that

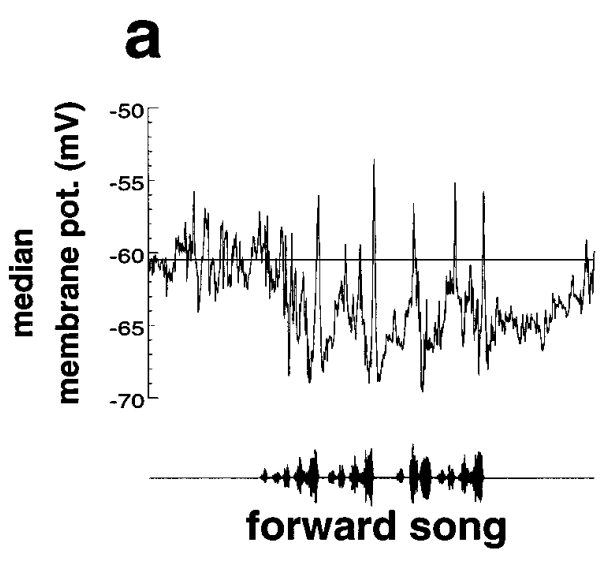

b
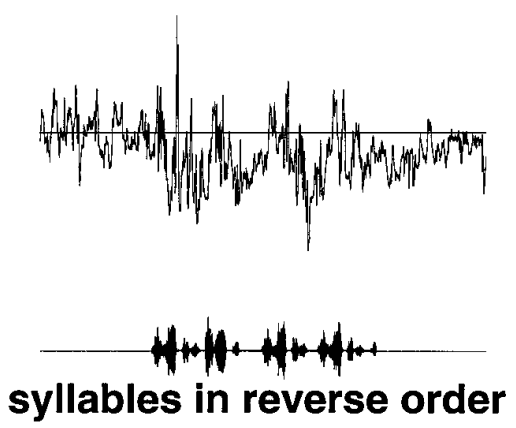

C
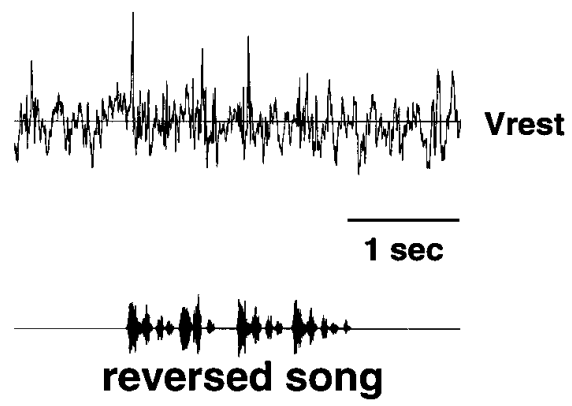

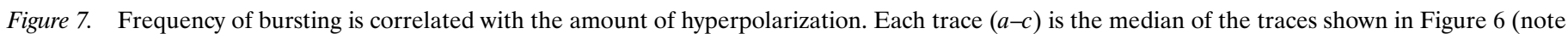

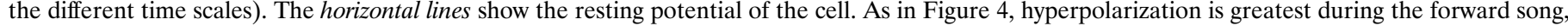
less when the syllables are presented in reverse order, and not present when the song is reversed. 
phasic song-specific

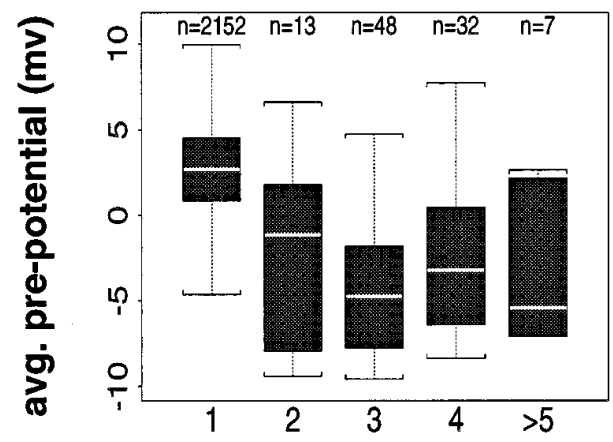

tonic song-specific

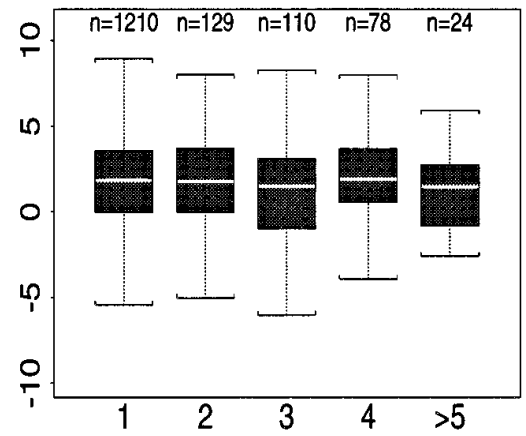

\#spikes in burst bursting non-auditory

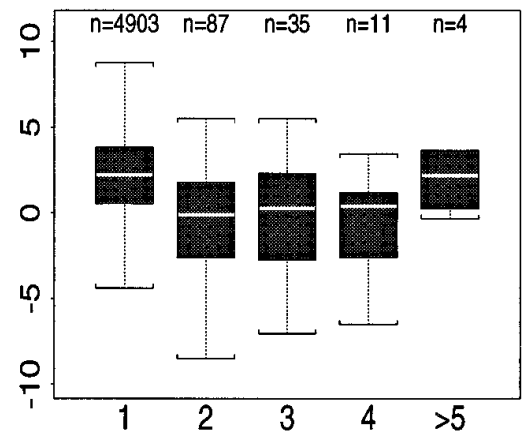

Figure 8. Correlation between bursting and level of prepotential. Each graph shows a plot of the distribution of prepotentials for single spikes and spike bursts containing the listed number of action potentials. Each box shows the middle half of each set of prepotentials. The horizontal line inside each box shows the median. The outer lines indicate $99 \%$ of the data range. Phasic song-specific cells showed significant hyperpolarization before each spike burst (left). Song-specific cells that were tonically excited also showed spike bursting (e.g., Fig. 1), but there was no significant change in the prepotential as a function of the number of spikes in a burst (middle). Nonauditory cells that generated spontaneous spike bursts, which were similar in appearance to those of the phasic cells, also showed significant previous hyperpolarization compared with the prepotential for a single spike (right).

some auditory neurons project to RA, which would be expected from physiological evidence that RA contains auditory neurons via inputs from HVc (Doupe and Konishi, 1991; Vicario and Yohay, 1993).

The evidence that song-specific cells can project to area $\mathrm{X}$ further supports the hypothesis that these cells are involved in learning. Area $\mathrm{X}$ is part of a series of song nuclei in the anterior forebrain that forms an additional pathway connecting HVc and RA. Lesions of area X do not affect song production in adults (Nottebohm et al., 1976), but lesions of the same area in young birds during the sensorimotor period (when the bird is learning to produce vocalizations) do prevent the development of normal song production (Bottjer et al., 1984; Scharff and Nottebohm, 1991). Song-specific cells are present in area $\mathrm{X}$ in adults and, like those in $\mathrm{HVc}$, emerge during vocal learning (Doupe and Konishi, 1992). These neurons are well situated to provide an auditory feedback signal to RA, and the song-specific cells in HVc could represent the first stage in the formation of this complicated circuitry.

\section{a}

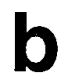

uluh
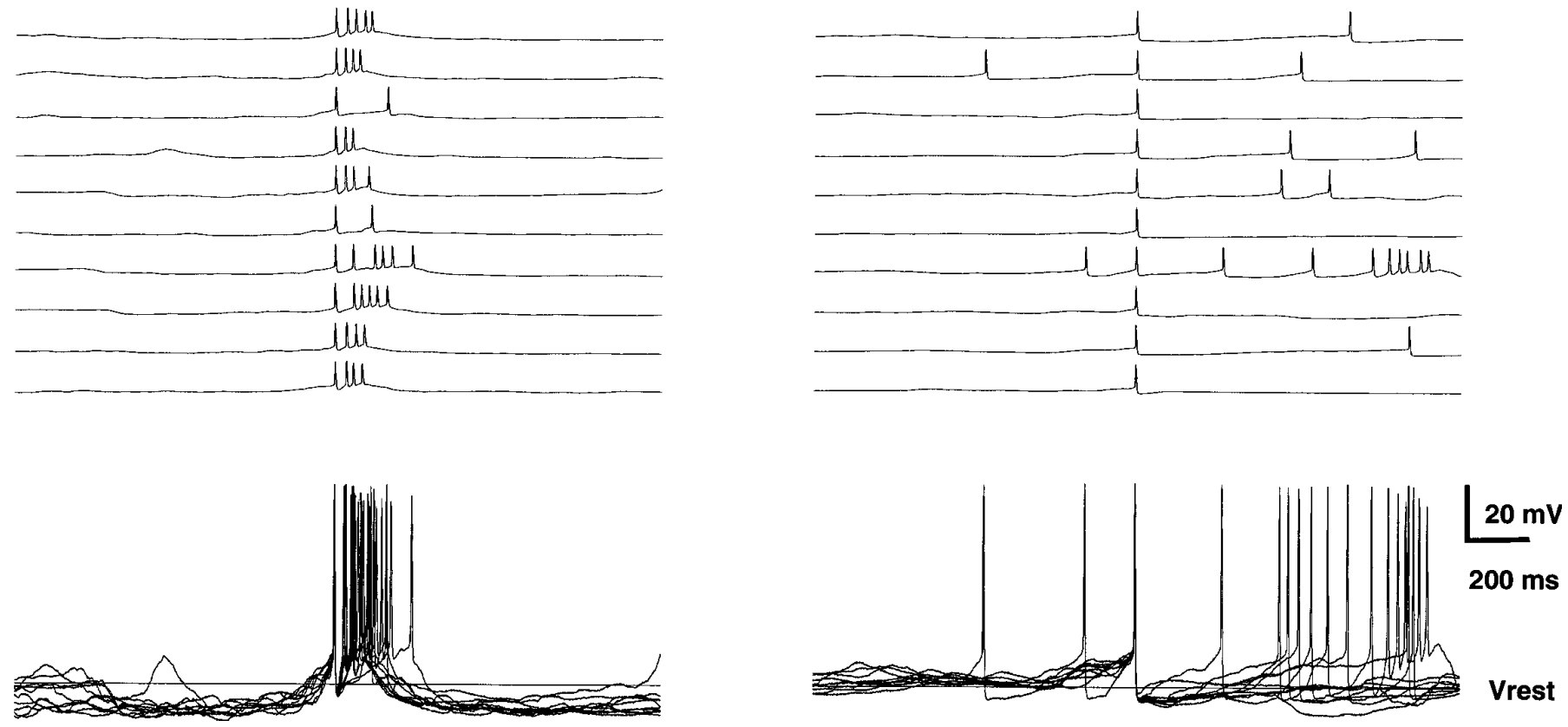

Figure 9. Examples of action potentials after low and high prepotentials. The action potentials in response to auditory stimuli (during stimulus and background periods) were sorted according to the average potential $50 \mathrm{msec}$ before each single spike or the first spike in each burst. Spikes with the lowest prepotential usually were followed by a burst of action potentials $(a)$; spikes with the highest prepotential were not $(b)$. 
a
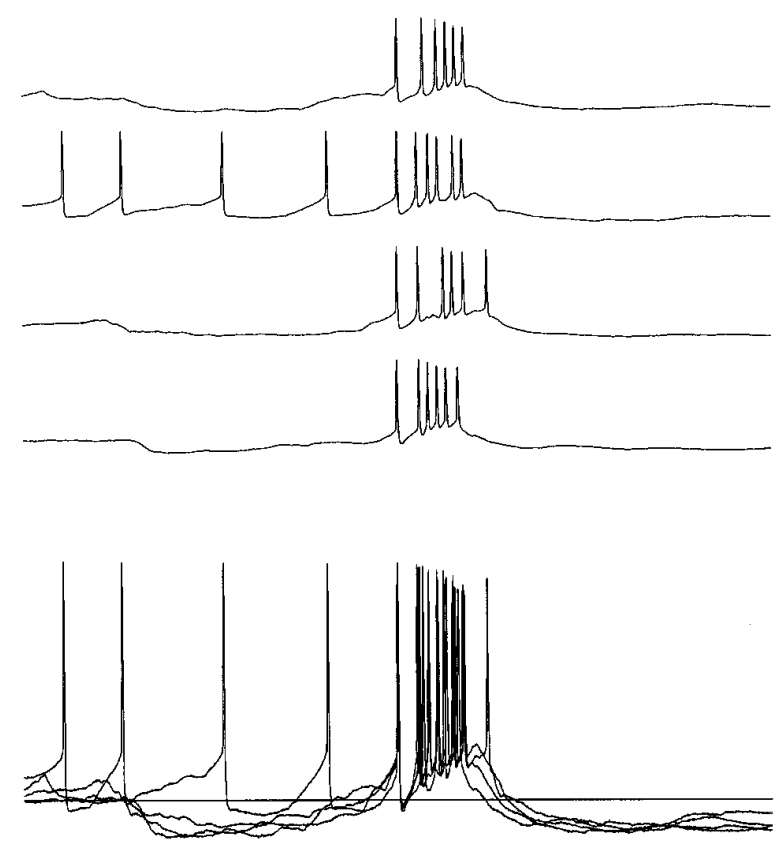

b
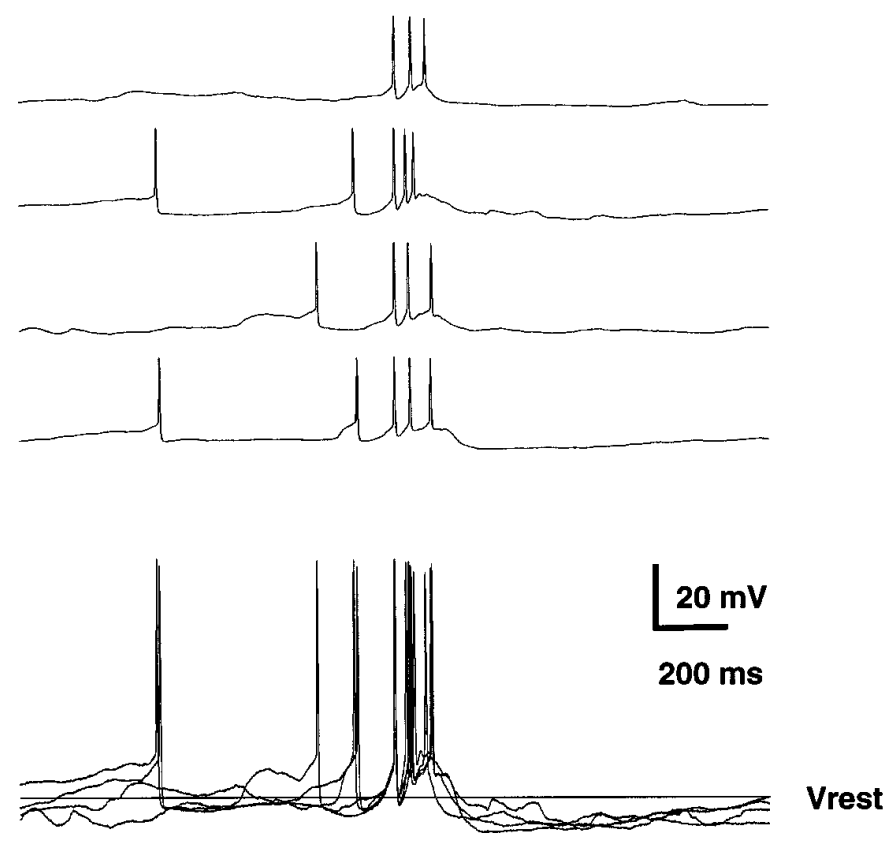

Figure 10. Examples of membrane potentials before different action potential bursts. The data shown here were extracted from the same stimulus and background periods that were used in the previous figure. In this analysis, a group of spikes was classified as a burst if the first two spikes were $<8$ msec apart. Each burst was sorted according to the number of spikes in the $30 \mathrm{msec}$ window after the first spike. Bursts with a greater number of spikes (a) tended to be preceded by a lower prepotential than bursts with fewer spikes $(b)$.

In many songbird species, including zebra finches, females sing little or not at all. Thus, it is interesting that the morphology of the song-specific cell in Figure $11 a$ is most like the thick dendrite class (based on Golgi staining), which is sexually dimorphic in canaries (Nixdorf et al., 1989).

\section{Song specificity}

A basic property of song-specific cells is a greater response to forward song compared with other songs. This would arise if the song-specific cells were integrating the output of neurons that already show some preference for forward song. Such neurons are known to be present in field L (Margoliash, 1986; Lewicki and Arthur, 1995), a group of auditory forebrain areas afferent to HVc. Part of the selectivity of these neurons can be accounted for by their sensitivity to the frequency modulation (FM) direction (Bonke et al., 1979; Leppelsack, 1983; Müller and Leppelsack, 1985; Hose et al., 1987; Knipschild et al., 1992). Downward FM is a prominent feature in the syllables of zebra finch song. Because reversing the song changes downward FM to upward FM, HVc cells could achieve some specificity for song by integrating the output of field L neurons that are sensitive to downward FM. This hypothesis is consistent with the intracellular observations of song-specific cells that were neither excited nor inhibited by reverse song. This may represent a first step in the generation of the more complex response properties.

Song-specific neurons also are sensitive to the temporal order of the syllables in autogenous song, which cannot be explained by sensitivity to spectral cues or FM. Furthermore, song-specific cells can integrate temporal information over periods lasting several hundred milliseconds (Margoliash, 1983; Margoliash and Fortune, 1992). Extracellular studies indicate that these properties do not exist in field $\mathrm{L}$ and that auditory selectivity undergoes additional refinement in HVc (Lewicki and Arthur, 1995).

The intracellular data presented here suggest many new mechanisms that could subserve this refinement. The long-lasting hyperpolarization during forward song provides a mechanism for preserving auditory context. Hyperpolarization also may be a prerequisite for burst firing, which could serve as a code for temporal events. Burst firing has been shown to be important in cells that are sensitive to combination of syllables in the correct temporal order (Lewicki and Konishi, 1995). The extensive intrinsic projections of HVc cells (Katz and Gurney, 1981; Fortune and Margoliash, 1995) allow for many possible neural circuits and a wide range of response properties.

These data are not sufficient in number to determine whether phasic and tonic responses represent distinct classes of songspecific cells. Extracellular studies may provide more plentiful data to address this question, but the results here suggest that data from extracellular studies of song-specific cells must be interpreted with caution. The intracellular data show that for some of the song-specific cells, the action potential shape during a burst can change dramatically, sometimes showing attenuation of the height as much as $50 \%$. This property makes bursting neurons difficult to study with conventional extracellular methods, because it is difficult to achieve single unit isolation. Thus, extracellular studies may be biased against these types of cells.

\section{Concluding remarks}

Song-specific neurons have some of the most complex auditory tuning properties yet discovered. The intracellular recordings of song-specific cells presented here have provided new insights into mechanisms underlying this selectivity. These mechanisms also 

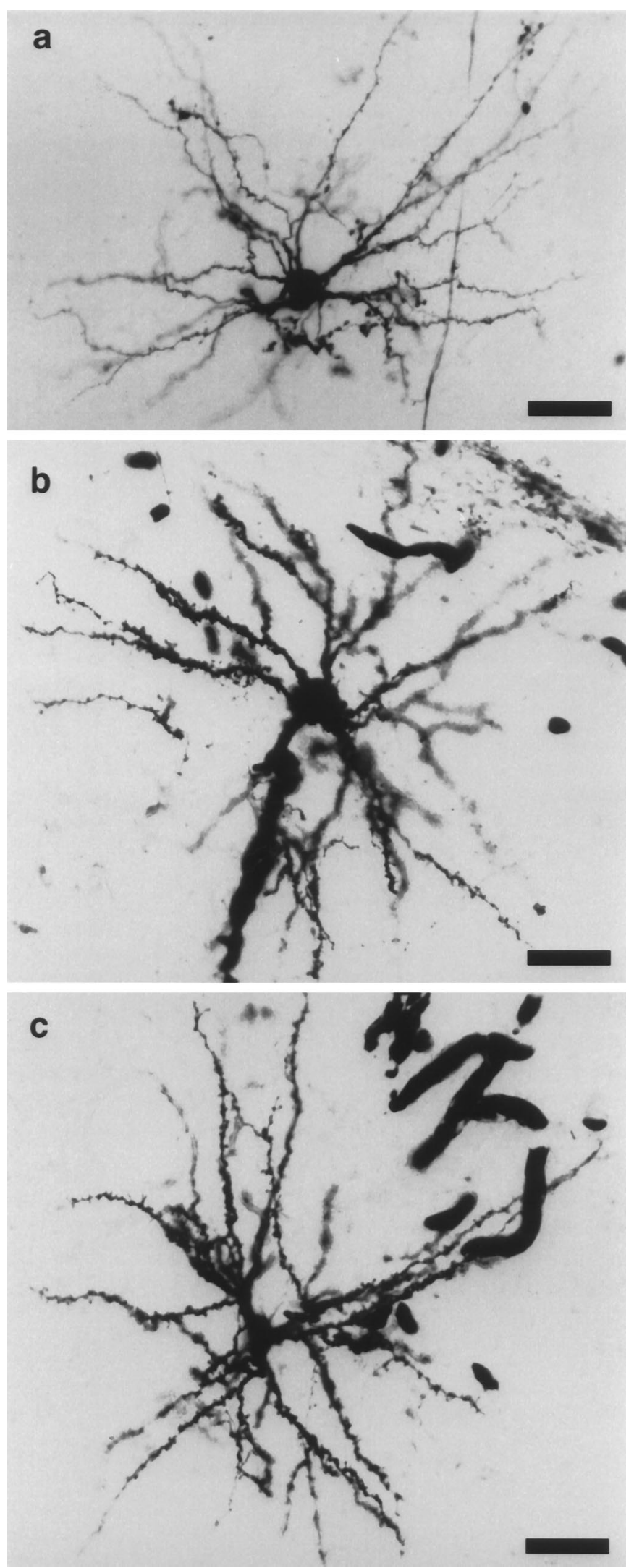

Figure 11. Photomicrographs of avidin-horseradish peroxidase-stained neurons. $a$, A song-specific HVc cell (data shown in Fig. 2); the axon of this cell could be traced to area X. $b, c$, Two nonauditory HVc cells; both cells had clear projections to RA. All scale bars, $20 \mu \mathrm{m}$. may subserve complex auditory neurons observed in other systems, such as the cat (Weinberger and McKenna, 1988; McKenna et al., 1989), squirrel monkey (Wollberg and Newman, 1972; Newman and Wollberg, 1973; Glass and Wollberg, 1983), and rhesus monkey (Rauschecker et al., 1995). The temporal pattern of the response of song-specific cells can be highly regular, even at the level of the subthreshold membrane potential. It remains to be seen whether the presence of such precise timing reflects that of afferent cells or whether it is refined by the circuitry in HVc. Precise timing is correlated with phasic bursting, and it is thus possible that phasic bursting and its associated hyperpolarization subserve a neural code utilizing spike timing. Such a code has obvious utility in the context of song learning and production.

\section{REFERENCES}

Bonke D, Scheich H, Langner G (1979) Responsiveness of units in the auditory neostriatum of the guinea fowl (Numida meleagris) to speciesspecific calls and synthetic stimuli. I. Tonotopy and functional zones of field L. J Comp Physiol [A] 132:243-255.

Bottjer SW, Miesner EA, Arnold AP (1984) Forebrain lesions disrupt development but not maintenance of song in passerine birds. Science 224:901-903.

Doupe AJ, Konishi M (1991) Song-selective auditory circuits in the vocal control system of the zebra finch. Proc Natl Acad Sci USA 88:11339-11343.

Doupe AJ, Konishi M (1992) Song-selective auditory neurons emerge during vocal learning in the zebra finch. Soc Neurosci Abstr 18:527.

Fortune ES, Margoliash D (1994) In vivo characterization of identified HVc neurons in the zebra finch. Soc Neurosci Abstr 20:165.

Fortune ES, Margoliash D (1995) Parallel pathways and convergence onto HVc and adjacent neostriatum of adult zebra finches (Taeniopygia guttata). J Comp Neurol 360:413-441.

Glass I, Wollberg Z (1983) Auditory-cortex responses to sequences of normal and reversed squirrel-monkey vocalizations. Brain Behav Evol 22:13-21.

Hose B, Langner G, Scheich H (1987) Topographic representation of periodicities in the forebrain of the myna bird: one map for pitch and rhythm. Brain Res 422:367-373.

Jahnsen H, Llinas R (1984) Electrophysiological properties of guinea-pig thalamic neurons: an in vitro study. J Physiol (Lond) 349:105-226.

Katz LC, Gurney ME (1981) Auditory responses in the zebra finch's motor system for song. Brain Res 211:192-197.

Kelley DB, Nottebohm F (1979) Projections of a telencephalic auditory nucleus-field L-in the canary. J Comp Neurol 183:455-470.

Kirkwood A, Dudek SM, Gold JT, Aizenman CD, Bear MF (1993) Common forms of synaptic plasticity in the hippocampus and neocortex in vitro. Science 260:1518-1521.

Knipschild M, Dorrscheidt GJ, Rubsamen R (1992) Setting complex tasks to single units in the avian auditory forebrain. I. Processing of complex artificial stimuli. Hear Res 57:216-230.

Konishi M (1965) The role of auditory feedback in the control of vocalization in the white-crowned sparrow. Z Tierpsychol 22:771-783.

Kubota M, Saito N (1991) Sodium-dependent and calcium-dependent conductances of neurons in the zebra finch hyperstriatum-ventrale pars caudale in vitro. J Physiol (Lond) 440:131-142.

Larson J, Wong D, Lynch G (1986) Patterned stimulation at the theta frequency is optimal for the induction of hippocampal long-term potentiation. Brain Res 368:347-350.

Leppelsack HJ (1983) Analysis of song in the auditory pathway of songbirds. In: Advances in vertebrate neuroethology (Ewert JP, ed), pp 783-800. New York: Plenum.

Lewicki MS, Arthur BJ (1995) Sensitivity to auditory temporal context increases significantly from field L to HVc. Soc Neurosci Abstr 21:958.

Lewicki MS, Konishi M (1995) Mechanisms underlying the sensitivity of songbird forebrain neurons to temporal order. Proc Natl Acad Sci USA 92:5582-5586.

Mainen ZF, Sejnowski TJ (1995) Reliability of spike timing in neocortical neurons. Science 268:1503-1506.

Malenka RC (1994) Synaptic plasticity in the hippocampus: LTP and LTD. Cell 78:535-538. 
Margoliash D (1983) Acoustic parameters underlying the responses of song-specific neurons in the white-crowned sparrow. J Neurosci 3:1039-1057.

Margoliash D (1986) Preference for autogenous song by auditory neurons in a song system nucleus of the white-crowned sparrow. J Neurosci 6:1643-1661.

Margoliash D, Fortune ES (1992) Temporal and harmonic combinationsensitive neurons in the zebra finch HVc. J Neurosci 12:4309-4326.

Margoliash D, Konishi M (1985) Auditory representation of autogenous song in the song system of white-crowned sparrows. Proc Natl Acad Sci USA 82:5997-6000.

McCasland JS (1987) Neuronal control of bird song production. J Neurosci 7:23-39.

McKenna TM, Weinberger NM, Diamond DM (1989) Responses of single auditory cortical-neurons to tone sequences. Brain Res 481:142-153.

Mooney R (1992) Synaptic basis for developmental plasticity in a birdsong nucleus. J Neurosci 12:2464-2477.

Mooney R, Konishi M (1991) Two distinct inputs to an avian song nucleus activate different glutamate receptor subtypes on individual neurons. Proc Natl Acad Sci USA 88:4075-4079.

Müller CM, Leppelsack HJ (1985) Feature extraction and tonotopic organization in the avian forebrain. Exp Brain Res 59:587-599.

Newman JD, Wollberg Z (1973) Multiple coding of species-specific vocalizations in the auditory cortex of squirrel monkeys. Brain Res 54:287-304.

Nixdorf BE, Davis SS, DeVoogd TJ (1989) Morphology of Golgiimpregnated neurons in hyperstriatum ventralis, pars caudalis in adult male and female canaries. J Comp Neurol 284:337-349.

Nottebohm F, Stokes TM, Leonard CM (1976) Central control of song in the canary, Serinus canarius. J Comp Neurol 165:457-486.

Rauschecker JP, Tian B, Hauser M (1995) Processing of complex sounds in the macaque nonprimary auditory-cortex. Science 268:111-114.
Scharff C, Nottebohm F (1991) A comparative study of the behavior deficits following lesions of various parts of the zebra finch song system: implications for vocal learning. J Neurosci 11:2896-2913.

Schmidt MF, Perkel DJ (1995) Characterization of slow inhibitory potentials in nucleus HVc of adult male zebra finches. Soc Neurosci Abstr 21:958.

Steriade M, Deschenes M (1984) The thalamus as a neuronal oscillator. Brain Res Rev 8:1-63.

Sutter ML, Margoliash D (1994) Global synchronous response to autogenous song in zebra finch HVc. J Neurophysiol 72:2105-2123.

Vates GE, Broome BM, Mello CV, Nottebohm F (1996) Auditory pathways of caudal telencephalon and their relation to the song system of adult male zebra finches (Taenopygia gutta). J Comp Neurol 366:613642

Vicario DS, Yohay KH (1993) Song-selective auditory input to a forebrain vocal control nucleus in the zebra finch. J Neurobiol 24:488-505.

Volman SF (1993) Development of neural selectivity for birdsong during vocal learning. J Neurosci 13:4737-4747.

Vu ET, Lewicki MS (1994) Intrinsic interactions between zebra finch HVc neurons involve NMDA-receptor mediated activation. Soc Neurosci Abstr 20:166.

Vu ET, Mazurek ME, Kuo YC (1994) Identification of a forebrain motor programming network for the learned song of zebra finches. J Neurosci 14:6924-6934

Weinberger NM, McKenna TM (1988) Sensitivity of single neurons in auditory cortex to contour: toward a neurophysiology of music perception. Music Perception 5:355-390.

Wollberg Z, Newman JD (1972) Auditory cortex of squirrel monkey: response patterns of single cells to species-specific vocalizations. Science 175:212-214.

Yu AC, Margoliash D (1995) Function hierarchy defined by single units in singing birds: HVc represents syllables and RA represents notes. Soc Neurosci Abstr 21:958. 